\title{
Application of a Novel Brain Arteriovenous Malformation Endovascular Grading Scale for Transarterial Embolization
}

\author{
D.L. Bell, T.M. Leslie-Mazwi, A.J. Yoo, J.D. Rabinov, W.E. Butler, J.E. Bell, and J.A. Hirsch
}

\begin{abstract}
BACKGROUND AND PURPOSE: The advent of modern neuroendovascular techniques has highlighted the need for a simple, effective, and reliable brain arteriovenous malformation endovascular grading scale. A novel scale of this type has recently been described. It incorporates the number of feeding arteries, eloquence, and the presence of an arteriovenous fistula component. Our aim is to assess the validity of this grading scale.
\end{abstract}

MATERIALS AND METHODS: We retrospectively reviewed all suspected brain arteriovenous malformations at Massachusetts General Hospital from 2005 to 2013, identifying 126 patients who met the inclusion criteria. Spearman correlations between endovascular and Spetzler-Martin grading scales and long-term outcomes were performed. Median endovascular grades were compared between treatment modalities and endovascular outcomes. Binary regression analysis was performed with major endovascular complications as a dichotomized dependent variable. Intraclass correlation coefficients were calculated for interobserver reliability of the endovascular grading scale.

RESULTS: A significant Spearman correlation between the endovascular grade and the Spetzler-Martin grade was demonstrated $(\rho=0.5$, $P<.01)$. Differences in the median endovascular grades between the endovascular cure (median $=2)$ and endovascular complication groups (median $=4)(P<.05)$ and between the endovascular cure and successful multimodal treatment groups $($ median $=3)(P<.05)$ were demonstrated. The endovascular grade was the only independent predictor of complications $(\mathrm{OR}=0.5, P<.01)$. The intraclass correlation coefficient of the endovascular grade was $0.71(P<.01)$.

CONCLUSIONS: Validation of a brain arteriovenous malformation endovascular grading scale demonstrated that endovascular grades of $\leq$ II were associated with endovascular cure, while endovascular grades of $\leq$ III were associated with multimodal cure or significant lesion reduction and favorable outcome. The endovascular grade provides useful information to refine risk stratification for endovascular and multimodal treatment.

ABBREVIATIONS: $A$ RUBA $=$ A Randomized Trial of Unruptured Brain Arteriovenous Malformations; $b$ AVM $=$ brain arteriovenous malformation; SIVMS $=$ Scottish Intracranial Vascular Malformation Study

S ince the first description of ethylene-vinyl alcohol copolymer as a liquid embolic agent for brain arteriovenous malformations (bAVMs) by Taki et $\mathrm{al}^{1}$ in 1990 and its regulatory approval in the United States in 2005, the application and proficiency of its use in the treatment of bAVMs has steadily evolved and improved. Dissolved in dimethyl-sulfoxide and known commer-

Received October 15, 2014; accepted after revision January 2, 2015.

From the Department of Radiology, Neuroendovascular Program (D.L.B., A.J.Y., J.D.R., J.A.H.), Department of Neurology and Radiology, Neurocritical Care and Neuroendovascular Program (T.M.L.-M.), and Department of Neurosurgery, Vascular Center (W.E.B.), Massachusetts General Hospital, Boston, Massachusetts; Harvard Medical School (D.L.B., T.M.L.-M., J.D.R., W.E.B., J.A.H.), Boston, Massachusetts; and Texas Stroke Institute (A.J.Y.), Plano, Texas.

Please address correspondence to Donnie L. Bell, MD, Massachussetts General Hospital, GRB 241, Boston, MA 02115; e-mail: d.l.bell.jr@gmail.com

http://dx.doi.org/10.3174/ajnr.A4286 cially as Onyx (Covidien, Irvine, California), the agent has expanded from its initial role as a palliative or presurgical/ preradiosurgical embolization agent to a stand-alone curative agent in selected cases. In the most recent large Onyx transarterial embolization series that included 350 patients, the rate of curative, durable Onyx embolization was $51 \%$, with $7.1 \%$ permanent morbidity and $1.4 \%$ mortality rates. ${ }^{2}$ Additionally, in complex lesions, particularly ruptured, deep, and/or lesions with arterial feeders en passage, transvenous Onyx embolization has been successful. $^{3-5}$

With expanded capability, appropriate patient selection becomes paramount. This was recently highlighted in the results of the controversial A Randomized Trial of Unruptured Brain Arteriovenous Malformations (ARUBA), which demonstrated a 3 -fold increased morbidity and mortality from multimodal inter- 
Table 1: Endovascular grading scale ${ }^{\mathrm{a}}$

Feature and Value

Points

\begin{tabular}{ll}
\hline No. of feeding $^{\text {arteries }}{ }^{\mathrm{b}}$ & 1 \\
$<3$ & 2 \\
$\geq 3,<6$ & 3 \\
$\geq 6$ & \\
Eloquence of adjacent areas & 0 \\
$\quad$ Noneloquent & 1 \\
$\quad$ Eloquent & \\
Presence of arteriovenous fistula component & \\
No AVF & 0 \\
AVF & 1
\end{tabular}

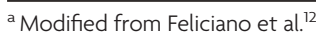

${ }^{\mathrm{b}}$ An arterial feeder was defined as a separate arterial pedicle or a pedicle arising $\geq 1.5$ $\mathrm{cm}$ from another arterial pedicle. En passage arterial feeders were given a score of 3 . ${ }^{c}$ The presence of an arteriovenous fistula was determined by criteria described by Yuki et $\mathrm{al}^{13}$ including an abnormally dilated feeding artery, a direct arteriovenous connection to a dilated venous component or varix, the absence of a plexiform component between the 2 structures, and a diameter of the feeding artery more than twice as large as that of the arteries supplying the comparable areas not supplying the AVM (eg, the corresponding contralateral cerebral artery) or feeding artery diameter of $>2 \mathrm{~mm}$.

ventional therapy as opposed to medical management $(30.7 \%$ versus $10.1 \%$, respectively). Notably, ARUBA was a pooled analysis incorporating bAVMs of varying Spetzler-Martin grades, limiting the applicability of the trial results to specific lesion types; additionally, $32 \%$ of the patients were treated with stand-alone embolization without standardized selection criteria. ${ }^{6,7}$ The findings of ARUBA were corroborated by the Scottish Intracranial Vascular Malformation Study (SIVMS), in which conservative management was associated with lower progression to the primary outcome, death, or sustained morbidity, with a hazard ratio of $0.6{ }^{8}$ In a similar fashion to ARUBA however, SIVMS was a pooled analysis of heterogeneous bAVMs, with $21.4 \%$ of patients treated with stand-alone embolization without standardized selection criteria. ${ }^{8,9}$

Although, the Spetzler-Martin and radiosurgical grading scales are validated means of predicting the safety and efficacy of surgery and radiosurgery respectively, application to endovascular or multimodal treatment (embolization plus radiosurgery or surgery) may be suboptimal as these grading schemes may not be sensitive to the procedural risk unique to transarterial embolization. ${ }^{10,11}$ No simple and effective endovascular grading scale for predicting procedural morbidity and/or mortality has been widely accepted. In 2011, Feliciano et al ${ }^{12}$ performed a literature review of the factors associated with outcomes in bAVM transarterial embolization and developed an endovascular grading scale incorporating the number of feeding arteries, eloquence, and the presence of an arteriovenous fistula component based on this review and their clinical experience. While intriguing due to its simplicity and incorporation of factors thought to predict transarterial embolization safety and efficacy, no criteria defining bAVMs amenable to endovascular treatment were defined and the grading scale was not validated. We aimed to refine this scale (Table 1) and apply it retrospectively to our bAVM database to assess its validity.

\section{MATERIALS AND METHODS Study Population}

We performed a retrospective analysis of the clinical data and imaging from 270 patients suspected of having ruptured or un- ruptured bAVMs presenting to the Massachusetts General Hospital from 2005 to 2013. Institutional review board approval for accessing these records for clinical research purposes was obtained from Massachusetts General Hospital. Onyx was the primary agent used during this study. Lesions were initially treated with transarterial embolization using Onyx for palliation or presurgical/preradiosurgical embolization; yet following the evolution of Onyx embolization techniques, we pursued the primary intention of endovascular cure in selected cases.

Patients were included in the study if they had a bAVM initially diagnosed and subsequently treated at Massachusetts General Hospital, with available clinical data and CT, MR imaging, or catheter digital subtraction cerebral angiography images in the electronic medical record. We excluded patients under the following circumstances: having incomplete clinical or imaging data; being lost to follow-up; lesions previously treated, treated at a different institution, that underwent autothrombosis, that were observed and medically managed, with only feeding or intranidal aneurysms treated, and that were deemed not bAVMs on catheter digital subtraction cerebral angiography. This left 126 patients for analysis.

\section{Data Collection}

Data collected from the electronic medical record included but was not limited to age, sex, presentation (hemorrhage, seizure, neurologic deficit, incidental finding), treatment technique (surgical, radiosurgical, endovascular, or multimodal — embolization followed by surgery or radiosurgery), modified Rankin scale (at presentation, following treatment, and at $\geq 90$ days), number of arterial feeders, presence of deep arterial feeders, arteriovenous fistula component, feeding artery or intranidal aneurysms, lesion volume, Spetzler-Martin grade, radiosurgical grade, and endovascular grade. Initial digital subtraction cerebral angiography images before any treatment were used for analysis. The images were independently reviewed by 2 neuroendovascular specialists (D.L.B., T.M.L.-M.), who assigned endovascular grades blinded to eventual treatment technique and outcomes. In lesions bordering eloquent areas and when functional imaging was performed, the functional imaging was used in determining Spetzler-Martin and endovascular grades. Final scores were adjudicated by the 2 reviewers.

\section{Endovascular Grading Scale}

The published scale was modified for the study, to make the scale more granular (Table 1). ${ }^{12}$ An arterial feeder was defined as a unique arterial pedicle if it originated $\geq 1.5 \mathrm{~cm}$ from another arterial pedicle. Arterial feeders were categorized into 3 groups based on the number (ie, 1-2 feeders, 3-5 feeders, and $\geq 6$ feeders, scored 1, 2, and 3 points, respectively). Predominant en passage arterial feeders were given a maximal score of 3 . Eloquence (absence $=0$, presence $=1$ point) was defined as outlined in the original description from the Spetzler-Martin grading scale. ${ }^{10}$ The presence of an arteriovenous fistula component (absence $=0$, presence $=1$ point) was determined by criteria described by Yuki et al, ${ }^{13}$ including an abnormally dilated feeding artery, a direct arteriovenous connection to a dilated venous component or varix, the absence of a plexiform 


\begin{tabular}{|c|c|c|c|c|c|c|}
\hline & $\begin{array}{l}\text { All Patients } \\
(n=126)\end{array}$ & $\begin{array}{l}\text { Endovascular } \\
\qquad(n=8)\end{array}$ & $\begin{array}{l}\text { Radiotherapy }{ }^{\mathrm{a}} \\
\quad(n=39)\end{array}$ & $\begin{array}{l}\text { Surgical } \\
(n=44)\end{array}$ & $\begin{array}{l}\text { Multimodal }^{\mathrm{a}} \\
\quad(n=31)\end{array}$ & $\begin{array}{l}\text { Endovascular } \\
\text { Complications } \\
\quad(n=4)\end{array}$ \\
\hline Age (yr) (mean) (SD) & $44.9( \pm 16.9)$ & $42.8(16.9)$ & $45.6(17.2)$ & $48.7(16.3)$ & $37.9(14.7)$ & $41.3(20.5)$ \\
\hline Female sex (\%) & 52 & 50 & 53 & 63 & 32 & 25 \\
\hline Endovascular grade (median) (IQR) & $3(2-4)$ & $2(1.75-2)$ & $4(2-4)$ & $3(2-3)$ & $3(2-4)$ & $4(3.5-4.25)$ \\
\hline Spetzler-Martin grade (median) (IQR) & $2(1-3)$ & $1(1.5-2)$ & $3(2-3)$ & $1(1-2)$ & $3(2-4)$ & $3.5(3-4)$ \\
\hline bAVM volume (mL) (mean) (SD) & $9.8( \pm 17.2)$ & $2.1( \pm 1.8)$ & $9.7( \pm 13.4)$ & $1.7( \pm 2.4)$ & $17.4( \pm 19.2)$ & $54.1( \pm 45.2)$ \\
\hline No. of feeding arteries (score) (median) (IQR) & $2(2-3)$ & $1(1-1.25)$ & $3(2-3)$ & $3(1-3)$ & $2(2-3)$ & $3(2.75-3)$ \\
\hline AVF component (\%) & $9 \%$ & 0 & $10 \%$ & $2 \%$ & $9 \%$ & $25 \%$ \\
\hline Eloquent (\%) & $53 \%$ & $63 \%$ & $74 \%$ & $31 \%$ & $54 \%$ & $75 \%$ \\
\hline Final mRS score (median) (IQR) & $0(0-1)$ & $0(0-0)$ & $0(0-1)$ & $0(0-1)$ & $1(0-1)$ & $5(3.5-6)$ \\
\hline
\end{tabular}

Note:-IQR indicates interquartile range.

${ }^{a}$ Cured or significantly reduced lesions without complications (in the "Multimodal" category, this includes embolization plus radiosurgery).

component between the 2 structures, and a diameter of the feeding artery more than twice as large as that of the arteries supplying the comparable areas not supplying the bAVM (for example, the corresponding contralateral cerebral artery) or a feeding artery diameter of $>2 \mathrm{~mm}$. As these authors report and as described by Lasjaunias et al, ${ }^{14}$ bAVMs may demonstrate a complex arterial nidus accompanied by arteriovenous fistula components that require selective microcatheter digital subtraction cerebral angiography for reliable identification. Because selective microcatheter digital subtraction cerebral angiography was not routinely performed during diagnostic digital subtraction cerebral angiography for bAVMs, our sensitivity for the detection of this component was reduced.

\section{Treatment}

Endovascular. Using Onyx-compatible microcatheters, bAVM arterial pedicles were superselected following the standard technique. Microcatheter digital subtraction cerebral angiography was then performed to elucidate the relationship of the arterial pedicle to other arterial branches, resolve the angioarchitecture of the bAVM, and define the venous egress. Patients with predominant en passage arterial feeders were not treated with transarterial embolization via those pedicles. Using intermittent blank roadmaps, we administered Onyx slowly using a thumb-tapping technique to establish a plug, followed by nidal penetration, without interruption of the Onyx injection for $>2$ minutes. Neither a detachable tip nor dual-lumen balloon microcatheters were available and/or used during the study period.

Surgical. The head was positioned for optimal exposure of the lesion and placed in fixation, and stereotactic craniotomy was performed. Microsurgical obliteration of bAVMs was achieved with the aid of stereotactic neuronavigation for bAVM localization. In ruptured lesions, concomitant hematoma evacuation was performed when indicated. Following surgical obliteration of the lesion, immediate postoperative digital subtraction cerebral angiography was performed to confirm resection.

Radiosurgical. Three stainless steel skull fiducials were placed for localization. The Gill-Thomas-Cosman stereotactic head frame was applied in the usual fashion. Using the skull fiducials for radiographic reference, the bAVM volume was adjusted at the isocenter and proton radiation was delivered. Each portal was
Table 3: Treatment by Spetzler-Martin grade

\begin{tabular}{lccc}
\hline & $\begin{array}{c}\text { Grade I/II } \\
\text { Lesions } \\
(\boldsymbol{n}=71)\end{array}$ & $\begin{array}{c}\text { Grade III } \\
\text { Lesions } \\
(\boldsymbol{n}=\mathbf{3 7 )}\end{array}$ & $\begin{array}{c}\text { Grade IV/V } \\
\text { Lesions } \\
(\boldsymbol{n}=\mathbf{1 8})\end{array}$ \\
\hline Surgery & $58 \%$ & $8 \%$ & $0 \%$ \\
Endovascular & $12 \%$ & $5 \%$ & $12 \%$ \\
Radiosurgery & $15 \%$ & $54 \%$ & $44 \%$ \\
Multimodal $^{\mathrm{a}}$ & $15 \%$ & $32 \%$ & $44 \%$ \\
\hline
\end{tabular}

${ }^{a}$ Embolization followed by surgery or radiosurgery.

custom-collimated and back-edge-compensated to provide maximum conformality to the dose distribution.

\section{Follow-Up}

Standard follow-up imaging was performed per institutional protocol in all treated cases. For surgery and endovascular treatment, it was 3-month posttreatment catheter digital subtraction cerebral angiography. For radiosurgical treatment delayed noninvasive imaging, typically contrast-enhanced MR imaging was performed at 2 years from treatment and then catheter digital subtraction cerebral angiography was performed at 3 years to document bAVM status at that time. Cured lesions were considered those without residual arteriovenous shunting on catheter digital subtraction cerebral angiography. $\mathrm{A} \geq 50 \%$ reduction in the lesion volume on any imaging technique was considered significant. Successful multimodal treatment was any cured or significantly reduced lesion without complications.

\section{Statistical Analysis}

Univariate Spearman correlation was performed with the endovascular grading scale, Spetzler-Martin grading scale, and 90 days mRS. A 2-tailed Mann-Whitney $U$ test was performed to compare the median endovascular grades between the endovascular cure and the endovascular complication groups and between the endovascular cure and the successful multimodal treatment groups. Backward stepwise binary regression analysis was performed with major endovascular complication as the dichotomized dependent variable and the number of arterial feeders, eloquence, arteriovenous fistula component, Spetzler-Martin grade, and endovascular grade as independent variables. Interobserver reliability of the endovascular grading scale and its components were calculated with intraclass correlation coefficients using a 2-way random model of absolute agreement type. SPSS, Version 21 (IBM, Armonk, New York) was used for statistical analysis. 


\section{RESULTS}

Total cohort demographics and of the 4 comparison groups are presented in Table 2. Treatment by the Spetzler-Martin grade is presented in Table 3. Notably, 58\% of grade I/II lesions were treated with surgical resection; $54 \%$ and $32 \%$ of grade III lesions were treated with radiosurgery and multimodal treatment, respectively; and $88 \%$ of grade IV/V lesions were treated with an even split of radiosurgery and multimodal treatment. Stand-alone endovascular treatment was performed in $12 \%$ of grade I/II lesions, $5 \%$ of grade III lesions, and $12 \%$ of grade IV/V lesions. Of the 31 patients treated with multimodal therapy, $68 \%$ were treated with transarterial embolization followed by surgery, while $32 \%$ were treated with transarterial embolization followed by radiosurgery. In the entire cohort, $38 \%$ presented with intracranial hemorrhage secondary to the bAVM. The overall and endovascular (as a stand-alone treatment and as part of multimodal treatment) rates of complications resulting in major morbidity or death were $3.4 \%$ and $9.3 \%$, respectively.

A statistically significant Spearman correlation between the endovascular grade and Spetzler-Martin grade was demonstrated $(\rho=0.5, P<.01)$. No correlations between the Spetzler-Martin or endovascular grade and the long-term mRS were identified.

There were significant differences in the median endovascular grades between the endovascular cure group (median $=2$; interquartile range, 1.75-2) and the endovascular complication group (median $=4$; interquartile range, 3.5-4.25; Mann-Whitney $U$ test $=3.5, P<.05)$ and between the endovascular cure group and the successful multimodal treatment group (median $=3$, interquartile range, 2-4; Mann-Whitney $U$ test $=56.5, P<.05$ ).

Backward stepwise binary regression analysis demonstrated the endovascular grade as the only independent predictor of complications $(\mathrm{OR}=0.5, P<.01)$.

The interobserver reliability of the endovascular grade was good, with an intraclass correlation coefficient of $0.71(P<.01)$. The intraclass correlation coefficients of the number of feeding arteries, eloquence, and arteriovenous fistulous component were $0.66,0.61$, and 0.53 , respectively $(P<.01)$.

\section{DISCUSSION}

Application of an endovascular grading scale incorporating the number of feeding arteries (1-2, 1 point; $3-5,2$ points; $\geq 6,3$ points), eloquence $($ absence $=0$, presence $=1$ point), and the presence of an arteriovenous fistula component $($ absence $=0$, presence $=1$ point $)$ demonstrated that endovascular grades of $\leq$ II were associated with endovascular cure, while endovascular grades of $\leq$ III were associated with multimodal cure or significant reduction and favorable outcomes $(P<.05)$. Moreover, the endovascular grade was the only independent predictor of major endovascular complications, demonstrated good interobserver reliability with an intraclass correlation coefficient of $0.71(P<.01)$, and correlated with the Spetzler-Martin grade. Illustrative lesions can be seen in Figs 1 and 2.

As neuroendovascular proficiency continues to advance, refinement of bAVM risk stratification for endovascular treatment is imperative in ensuring favorable outcomes. The Spetzler-Martin grade has been adapted to predict morbidity associated with stand-alone embolization with long-term rates of neurologic deficits after embolization of $0 \%, 5 \%, 7 \%, 10 \%$, and $18 \%$, respectively, for Spetzler-
Martin grades I through V. ${ }^{15}$ However, in this study, $n$-butyl cyanoacrylate and coils were the primary agents as opposed to Onyx. The Spetzler-Martin grading scale has also been shown to correlate with outcomes in patients undergoing multimodal treatment. ${ }^{16,17}$ Nevertheless, it was created for surgical risk assessment, and because transarterial embolization of bAVMs aims to obliterate bAVMs via endovascular arterial occlusion and inflammatory changes leading to angionecrosis while carrying the risk of premature venous occlusion before complete devascularization of the lesion, the factors influencing the safety and efficacy of transarterial embolization likely differ from those governing the success of surgery. ${ }^{18}$

To our knowledge, this is the first validation of an endovascular grading scale for bAVMs for transarterial embolization since the advent of current neuroendovascular techniques. Previously, an endovascular grading scale was reported by Willinsky et $\mathrm{al}^{19}$ in 2001 for $\mathrm{bAVMs}$ of $<3 \mathrm{~cm}$; this scale included nidus type/size (fistula $=0 ;<1$ $\mathrm{cm}=1$ point $1-3 \mathrm{~cm}=2$ points), type of feeding arteries (cortical $=$ 0 , perforator or choroidal $=1$ point), number of feeding arteries ( single $=0$, multiple $=2$ points), and the number of draining veins (single $=0$, multiple $=1$ point). In 80 patients, the authors demonstrated a cure rate of $86 \%$ in grade $0-2$ lesions and suggested that this scale was a better predictor of endovascular cure, morbidity, and mortality than the Spetzler-Martin grade. A key differentiating factor between the grading scale presented by Willinsky et $\mathrm{al}^{19}$ and the endovascular grading scale presented here is the grading of arteriovenous fistula components. In the previous grading system, arteriovenous fistula components did not increase the endovascular grade as they do in the current grading system. Most interesting, 3 of 7 complications experienced by Willinsky et al were the result of venous penetration of the embolic agent, and whether an arteriovenous fistula component played a role in these outcomes might be questioned. An additional distinguishing factor is the incorporation of the number of draining veins that did not correlate with cure rates in their study, which is absent in the grading scale presented here. ${ }^{19}$ Finally, the prior scale was developed with the use of isobutyl cyanoacrylate and $n$-butyl cyanoacrylate as the liquid embolic agents. Isobutyl cyanoacrylate is no longer in use, and $n$-butyl cyanoacrylate is now typically an adjunctive endovascular agent or a primary agent in selected cases.

Since the report by Willinsky et $\mathrm{al},{ }^{19}$ Onyx has been introduced; neuroendovascular techniques have evolved; and myriad factors, including the Spetzler-Martin grade, periprocedural hemorrhage, deep venous drainage, number of embolized branches, age, absence of neurologic deficit, hemorrhagic presentation, small bAVM size, the presence of deep arterial feeders, eloquent location, the presence of arteriovenous fistula components, and venous penetration of embolic agents, have been associated with endovascular complications and unfavorable outcomes. ${ }^{15,19-23}$ Furthermore, adding to our understanding of the mechanisms underlying complications and their frequencies following bAVM transarterial embolization, Baharvahdat et $\mathrm{al}^{24}$ noted in their series of 846 cases primarily treated with Onyx that of the $11 \%$ of patients experiencing hemorrhagic complications, those complications could be divided into 2 categories: arterial perforation (48\%) and all others (52\%). Arterial perforation occurred during either microcatheter navigation or retrieval. Of the hemorrhagic complications not attributable to arterial perforation, premature venous deposition was an independent predictor of com- 

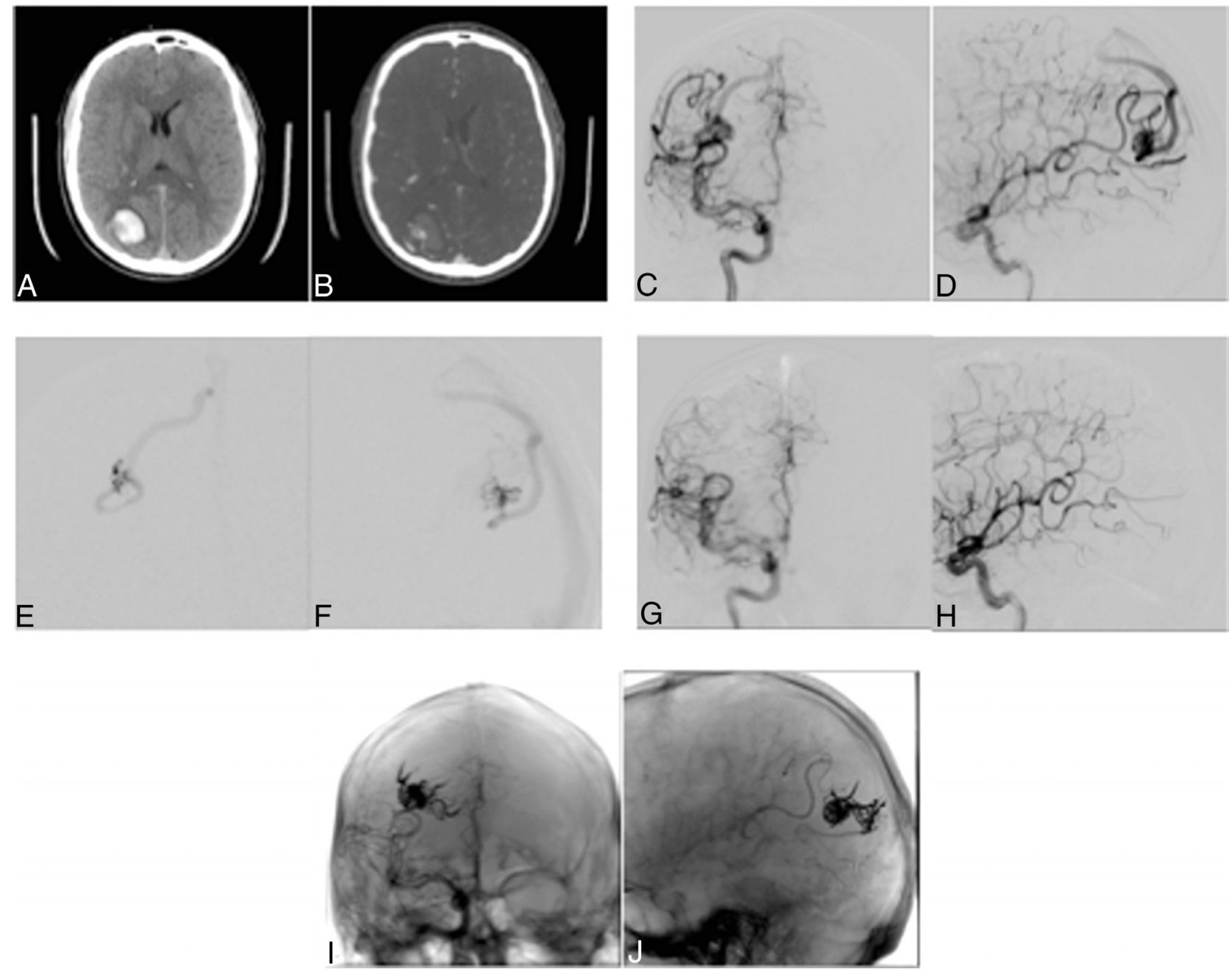

FIG 1. A 35-year-old man presenting with acute onset of dizziness and headache. $A$ and $B$, noncontrast head CT and head CTA demonstrate a 3.4- $\mathrm{cm}$ right parietal hematoma and a 1.9-cm bAVM, respectively. $C$ and D, Diagnostic right internal carotid artery anteroposterior and lateral angiograms demonstrate a Spetzler-Martin grade II (S1, El, V0) and endovascular grade II (A2, EO, F0) bAVM supplied via angular branches of the right middle cerebral artery (supplementary right posterior cerebral artery supply not shown) with superficial venous drainage via mildly dilated cortical veins. $E$ and $F$, Intraprocedural right middle cerebral artery angular branch microcatheter anteroposterior and lateral angiograms demonstrate a distinct plexiform nidus and no arteriovenous fistulous component. $G$ and $H$, Right internal carotid artery anteroposterior and lateral angiograms following Onyx embolization via the right middle cerebral artery angular branch demonstrate resolution of arteriovenous shunting (left vertebral artery injection also documented resolution of arteriovenous shunting from a supplementary right posterior cerebral artery supply, not shown). I and J, Skull anteroposterior and lateral radiographs demonstrate the Onyx cast; note penetration of the Onyx material into a posterior component supplied by the right posterior cerebral artery on the lateral projection. Final $m R S=0$. S indicates size; $E$, eloquence; $V$, vein; $A$, artery; $F$, fistula.

plications. Notably, complications did not correlate with increasing Spetzler-Martin grade. ${ }^{24}$ Intuitively then, increasing numbers of arterial feeders requiring multiple microcatheterizations and/or multistage procedures, the presence of an arteriovenous fistula component increasing the likelihood of premature venous embolization, and eloquent regions intolerant to insult are likely to be associated with complications and poor outcomes following transarterial embolization.

With the recent results of the ARUBA and SIVMS trials, patient selection for bAVM treatment will likely come under greater scrutiny. The limitations of these trials are well recognized, including the lack of standardized interventional arm treatment algorithms, selection bias, variable participating site characteristics, short follow-up period, the relatively small number of Spetzler-Martin grade I and II lesions treated with surgery, and the small sample size that limited subset analysis. ${ }^{7,9,25}$ Although the literature suggests that the Spet-
zler-Martin grading scale may be applicable to endovascular and multimodal treatment in some cases, discrepant lesions with low Spetzler-Martin grades and high endovascular grades might pose a challenge regarding patient selection. In our cohort, 3 of the 4 patients with major complications had an endovascular grade of $\geq 4$ with 1 lesion harboring an arteriovenous fistula component that was prematurely embolized, resulting in occlusive hyperemia and hemorrhagic venous infarction (Fig 2). Notably, in 2 of these 4 lesions, the endovascular grade was higher than the Spetzler-Martin grade, including the lesion in Fig 2. Given this finding, the endovascular grading scale may supplement Spetzler-Martin grades in lesions under consideration for endovascular and multimodal treatment, improving patient selection.

The interobserver agreement of the endovascular grading scale was good and approaching excellent, with an intraclass correlation coefficient of $0.71(P<.01)$. Interobserver variability largely 

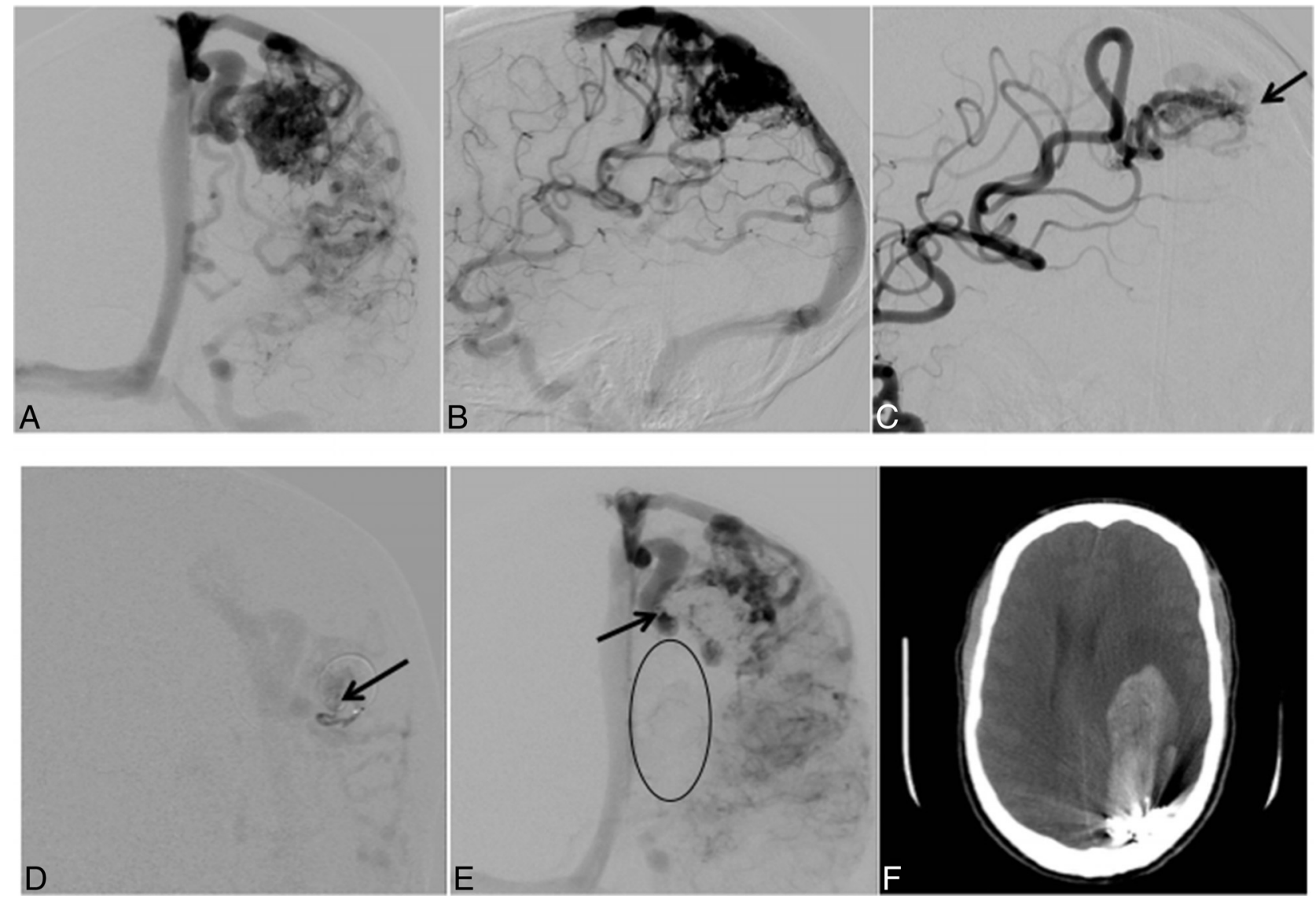

FIG 2. A 31-year-old man presenting with seizures found to have a 3.8-cm left frontoparietal bAVM, Spetzler-Martin grade III (S2, El, V0), and endovascular grade $\mathrm{V}(\mathrm{A} 3, \mathrm{El}, \mathrm{F})$. $A$ and $B$, Diagnostic left internal carotid anteroposterior and lateral angiograms demonstrate predominant arterial supply to the lesion via posterior frontal and parietal branches of the left middle cerebral artery with superficial venous drainage via moderately dilated cortical veins. Supplemental supply via the left anterior and posterior cerebral arteries was also present (not shown) C, Diagnostic left middle cerebral artery lateral magnification angiogram demonstrates a dilated angular branch $>2 \mathrm{~mm}$ with a direct venous connection consistent with an arteriovenous fistula component (arrow). D, Intraprocedural left middle cerebral artery angular branch superselective microcatheter injection oblique anteroposterior angiogram again demonstrates an arteriovenous fistula component (arrow). E, Following stage I Onyx embolization, the medial portion of the Onyx cast (Onyx subtracted, seen as absence of contrast enhancement within the previous nidus compared to image $A$ ) can be seen within a dilated medial cortical vein (arrow), and the previously seen dilated cortical veins coursing inferiorly in image $A$ are no longer visualized consistent with venous occlusion (ellipse).F, Postprocedural day 2 emergent noncontrast head CT performed for neurologic deterioration and a dilated left pupil demonstrates a large $3.8 \times 7.4 \mathrm{~cm}$ left frontal hemorrhage anterior to the Onyx cast with surrounding cerebral edema, causing moderate-to-severe rightward midline shift. The hematoma and residual bAVM underwent emergent surgical resection. Final mRS was 6 due to neurologic decline and ventilator-associated pneumonia. S indicates size; $E$, eloquence; $V$, vein; $A$, artery; $F$, fistula.

arose from discrepancies in the designation of arterial feeders en passage, determining eloquence in lesions bordering eloquent areas, and detection of arteriovenous fistula components. Variability in arterial feeders en passage and arteriovenous fistula components might be resolved by superselective catheterization in diagnostic digital subtraction cerebral angiography or at treatment in lesions preliminarily deemed amenable to transarterial embolization. A consensus agreement regarding areas bordering eloquent regions, overlapping eloquent and noneloquent lesions graded as eloquent, and/or performance of functional imaging or superselective provocative pharmacologic testing could be used in lesions in which eloquence is in question.

We did not demonstrate a correlation between Spetzler-Martin and endovascular grades with long-term outcomes. Potential explanations include patient selection (only $8 \%$ of lesions Spetzler-Martin grade III or higher were treated with surgery, and a large fraction, $72 \%$, of patients treated with transarterial embolization were treated as part of multimodal therapy), a low overall complication rate, and a small sample size.
While we have detailed criteria for an endovascular grading scale for transarterial embolization of bAVMs and demonstrated its utility and reliability, there are several limitations of our study. The small sample size and retrospective, single-center study design limit generalization. The lack of routine arterial pedicle superselective microcatheter digital subtraction cerebral angiography during diagnostic evaluation decreased the sensitivity for the detection of arterial feeders en passage and arteriovenous fistula components. Also the applicability of the endovascular grading scale to risk stratification for transvenous bAVM embolization and newer transarterial embolization methods, including the pressure cooker and the dual-lumen balloon microcatheter techniques, which may expand the types of bAVMs amenable to safe and effective endovascular treatment, cannot be assessed. Further, the endovascular grading scale does not incorporate hemodynamic data such as wall sheer stress and other flow-related parameters, which may identify bAVM components susceptible to hemorrhage or complications following transarterial embolization. Finally, despite our diligent search, there may have been occa- 
sional cases that were not identified and hence not analyzed. ${ }^{26-30}$ In the case of venous egress, a dichotomized variable for the number of venous egress pathways ( variations thereof, in addition to or supplanting the number of arterial feeders, might extend the applicability of this grading scale to the transvenous approach. Future studies evaluating the endovascular grading scale in a prospective manner are warranted.

\section{CONCLUSIONS}

A novel endovascular grading scale for bAVM transarterial embolization incorporating the number of feeding arteries (1-2, 1 point; $3-5,2$ points; $\geq 6,3$ points), eloquence (absence $=0$, presence $=1$ point), and the presence of an arteriovenous fistula component (absence $=0$, presence $=1$ point $)$ demonstrated that endovascular grades of $\leq$ II were associated with endovascular cure, while endovascular grades of $\leq$ III were associated with a multimodal cure or significant lesion reduction and favorable outcomes. Moreover, the endovascular grade was an independent predictor of major endovascular complications and demonstrated good interobserver reliability. The endovascular grading scale may supplement Spetzler-Martin grades to refine risk stratification for endovascular and multimodal bAVM treatment. Further validation is warranted.

\section{ACKNOWLEDGMENTS}

We thank Drs Johnny Pryor, Christopher Ogilvy, and Paul Chapman as clinical investigators who provided and cared for study patients.

Disclosures: Joshua A. Hirsch—UNRELATED: Consultancy: Medtronic, Comments: interventional spine; Stock/Stock Options: Intratech, Comments: developmentalstage interventional stroke company.

\section{REFERENCES}

1. Taki W, Yonekawa Y, Iwata H, et al. A new liquid material for embolization of arteriovenous malformations. AJNR Am J Neuroradiol 1990;11:163-68

2. Saatci I, Geyik S, Yavuz K, et al. Endovascular treatment of brain arteriovenous malformations with prolonged intranidal Onyx injection technique: long-term results in 350 consecutive patients with completed endovascular treatment course. J Neurosurg 2011;115:78-88

3. Albuquerque FC, Ducruet AF, Crowley RW, et al. Transvenous to arterial Onyx embolization. J Neurointerv Surg 2014;6:281-85

4. Consoli A, Renieri L, Nappini S, et al. Endovascular treatment of deep hemorrhagic brain arteriovenous malformations with transvenous Onyx embolization. AJNR Am J Neuroradiol 2013;34:1805-11

5. Nguyen TN, Chin LS, Souza R, et al. Transvenous embolization of a ruptured cerebral arteriovenous malformation with en-passage arterial supply: initial case report. J Neurointerv Surg 2010;2:150-52

6. Mohr JP, Parides MK, Stapf C, et al. Medical management with or without interventional therapy for unruptured brain arteriovenous malformations (ARUBA): a multicentre, non-blinded, randomised trial. Lancet 2014;383:614-21

7. Bambakidis NC, Cockroft KM, Hirsch JA, et al. The case against a randomized trial of unruptured brain arteriovenous malformations: misinterpretation of a flawed study. Stroke 2014;45:2808-10

8. Al-Shahi Salman R, White PM, Counsell CE, et al. Outcome after conservative management or intervention for unruptured brain arteriovenous malformations. JAMA 2014;311:1661-69

9. Zaidi HA, Russin JJ, Spetzler RF. Conservative management vs intervention for unruptured brain arteriovenous malformations. JAMA 2014;312:1058
10. Spetzler RF, Martin NA. A proposed grading system for arteriovenous malformations. J Neurosurg 1986;65:476-83

11. Pollock BE, Flickinger JC. A proposed radiosurgery-based grading system for arteriovenous malformations. J Neurosurg 2002;96:79-85

12. Feliciano C, de León-Berra R, Hernández-Gaitán MS, et al. A proposal for a new arteriovenous malformation grading scale for neuroendovascular procedures and literature review. $P$ R Heal Sci J 2011;29:117-20

13. Yuki I, Kim RH, Duckwiler G, et al. Treatment of brain arteriovenous malformations with high-flow arteriovenous fistulas: risk and complications associated with endovascular embolization in multimodality treatment: clinical article. J Neurosurg 2010;113:715-22

14. Lasjaunias P, Manelfe C, Chiu M. Angiographic architecture of intracranial vascular malformations and fistulas: pretherapeutic aspects. Neurosurg Rev 1986;9:253-63

15. Kim LJ, Albuquerque FC, Spetzler RF, et al. Postembolization neurological deficits in cerebral arteriovenous malformations: stratification by arteriovenous malformation grade. Neurosurgery 2006;59: 53-59; discussion 53-59

16. Deruty R, Pelissou-Guyotat I, Mottolese C, et al. Prognostic value of the Spetzler's grading system in a series of cerebral AVMs treated by a combined management. Acta Neurochir (Wien) 1994;131:169-75

17. Nataraj A, Mohamed MB, Gholkar A, et al. Multimodality treatment of cerebral arteriovenous malformations. World Neurosurg 2014;82:149-59

18. Jahan R, Murayama Y, Gobin YP, et al. Embolization of arteriovenous malformations with Onyx: clinicopathological experience in 23 patients. Neurosurgery 2001;48:984-95; discussion 995-97

19. Willinsky R, Goyal M, Terbrugge K, et al. Embolisation of small (<3 $\mathrm{cm})$ brain arteriovenous malformations: correlation of angiographic results to a proposed angioarchitecture grading system. Interv Neuroradiol 2001;7:19-27

20. Ledezma CJ, Hoh BL, Carter BS, et al. Complications of cerebral arteriovenous malformation embolization: multivariate analysis of predictive factors. Neurosurgery 2006;58:602-11; discussion 602-11

21. Gobin YP, Laurent A, Merienne L, et al. Treatment of brain arteriovenous malformations by embolization and radiosurgery. $J$ Neurosurg 1996;85:19-28

22. Hartmann A, Mast H, Mohr JP, et al. Determinants of staged endovascular and surgical treatment outcome of brain arteriovenous malformations. Stroke 2005;36:2431-35

23. Haw CS, terBrugge K, Willinsky R, et al. Complications of embolization of arteriovenous malformations of the brain. J Neurosurg 2006;104:226-32

24. Baharvahdat $H$, Blanc $\mathrm{R}$, Termechi R, et al. Hemorrhagic complications after endovascular treatment of cerebral arteriovenous malformations. AJNR Am J Neuroradiol 2014;35:978-83

25. Amin-Hanjani S, Albuquerque FC. Commentary: unruptured brain arteriovenous malformations-what a tangled web they weave. Neurosurgery 2014;75:195-96

26. Chapot R, Stracke P, Velasco A, et al. The pressure cooker technique for the treatment of brain AVMs. J Neuroradiol 2014;41:87-91

27. Illies T, Forkert ND, Ries T, et al. Classification of cerebral arteriovenous malformations and intranidal flow patterns by color-encoded 4D-hybrid-MRA. AJNR Am J Neuroradiol 2013;34:46-53

28. Ansari SA, Schnell S, Carroll T, et al. Intracranial 4D flow MRI: toward individualized assessment of arteriovenous malformation hemodynamics and treatment-induced changes. AJNR Am J Neuroradiol 2013;34:1922-28

29. Orozco LD, Luzardo GD, Buciuc RF. Transarterial balloon assisted Onyx embolization of pericallosal arteriovenous malformations. J Neurointerv Surg 2013;5:e18

30. Jagadeesan BD, Grigoryan M, Hassan AE, et al. Endovascular balloon-assisted embolization of intracranial and cervical arteriovenous malformations using dual-lumen coaxial balloon microcatheters and Onyx: initial experience. Neurosurgery 2013;73(2 suppl operative):ons 238-43; discussion ons 243 